10.1136/ebnurs-2012-100750

Department of Community Public Health, Johns Hopkins University School of Nursing, Baltimore, Maryland, USA

Correspondence to: Sarah J M Shaefer Department of Community Public Health, Johns Hopkins University School of Nursing, 525 N. Wolfe St., Room 419, Baltimore, MD 21205-2110, USA;

jshaefer@jhmi.edu

Systematic review and meta-analysis

\title{
Review finds that bed sharing increases risk of sudden infant death syndrome
}

\section{Sarah J M Shaefer}

Commentary on: Vennemann MM, Hense HW, Bajanowski T, et al. Bed sharing and the risk of sudden infant death syndrome: can we resolve the debate? J Pediatr 2012;160:44-8.e2.

\section{Implications for practice and research}

Practitioners should inform parents of higher sudden infant death syndrome risk because of bed sharing, especially for smokers and very young infants.

Parents should be encouraged to share a room with an infant, not a bed.

Additional case control studies are needed on all aspects of bed sharing.

Future research should explore parental decision making for sleep location and position.

\section{Context}

Sudden infant death syndrome (SIDS) remains the leading cause of infant mortality in USA and most developed countries. After studies in the late 1980s and early 1990s which identified the prone sleep position as a main risk for SIDS, campaigns to promote back sleeping were initiated, and the number of SIDS declined significantly. ${ }^{1}$ However, the question of risk related to bed sharing remains unresolved. Controversy remains over bed sharing recommendations, with some countries advising against bed sharing altogether, and others advising against it in certain circumstances. This study is a meta-analysis of case-control studies to provide a thorough review of available evidence on the risk of SIDS associated with bed sharing.

\section{Methods}

Two of the authors searched PubMed and Medline for the subjects 'sudden infant death syndrome,' 'sudden unexpected death,' and 'cot death' with 'bed sharing' or 'co-sleeping.' All studies published in any language after 1 January 1970 were considered. A total of 212 abstracts were identified. Inclusion criteria included adequate SIDS definition, autopsy, clear description of control selection and sufficient data to calculate OR and 95\% CIs. Eleven studies (USA-3, New Zealand-1, UK-2, Norway-1, Ireland-1, Germany-1) met the criteria. The sample consisted of 2464 cases and 6495 controls, of which 710 cases (28.8\%) and 863 controls (13.3\%) bed-shared.

\section{Findings}

The summary OR for bed sharing and SIDS was 2.89 (95\% CI 1.99 to 4.18). Despite some heterogeneity, all studies found an increased risk of SIDS in bed-sharing infants. Subgroup analyses for the studies reporting maternal smoking revealed an OR of 6.27 (95\% CI 3.94 to 9.99) for smoking and bed sharing. The OR was not significant for non-smokers and bed sharing. Infants less than 12 weeks had a significant risk for SIDS because of bed sharing (OR 10.37, 95\% CI 4.44 to 24.21), while the risk was not significant for older infants. Routine bed sharing was not significantly associated with SIDS (OR 1.43, 95\% CI 0.85 to 2.38), but the risk was significant for those who shared a bed on the night of death when bed sharing was not routine.

\section{Commentary}

This meta-analysis is comprehensive, provides international data, and consistent assessment of SIDS diagnosis. One limitation is that the study includes cases before and after the 'back to sleep' campaign. Previous findings have confirmed that smoking and bed sharing increases 
an infant's risk of SIDS. The data support the new recommendations by the American Academy of Pediatrics that have expanded the 'back to sleep' message to safe sleep environment. ${ }^{1}$ Maternal smoking and infant age under 12 weeks dramatically increase SIDS risk that is associated with bed sharing. Furthermore, some studies have suggested that SIDS risk is increased when there are multiple bed sharers, when the bed sharer has consumed alcohol or drugs or is overtired, or when an adult shares a sofa with an infant. However, Vennemann et al were unable to examine these factors in their analyses. While the issue of bed sharing and SIDS remains controversial, findings from this study suggest health messages that can be added to public awareness campaigns and infant care education for pregnant women. Nurses play a key role in disseminating these health messages via health education and role modelling safe sleep environment. ${ }^{2}$ The 'back to sleep' campaign success was in part due to a single message. The results of this meta-analysis suggest that the current message should be that infants ought to sleep alone in their own bed.
At a minimum, the authors recommend warning families against bed sharing when either parent smokes, when the infant is under 12 weeks of age, when the parent has consumed alcohol or drugs or when sleeping on sofas. To date, no single study has demonstrated a reduced risk of SIDS in bed sharing with infants. Finally, the fact that only 11 studies met the authors' inclusion criteria for this meta-analysis indicate the need for more research to identify the aspects of bed sharing that increase the risk for SIDS, as well as factors that interact with bed sharing to amplify risk.

\section{Competing interests None.}

\section{References}

1. Moon RY. SIDS and other sleep-related infant deaths: expansion of recommendations for a safe infant sleeping environment. Pediatrics 2011;128:e1341-67.

2. Shaefer SJ, Herman SE, Frank SJ, et al. Translating infant safe sleep evidence into nursing practice. J Obstet Gynecol Neonatal Nurs 2010;39:618-26. 